\title{
Abstract \\ Characteristics of the patients with coronary slow flow syndrome (syndrome y)
}

Bandara HGWAPL ${ }^{1}$, Kogulan $\mathrm{T}^{1}$, Kodithuwakku $\mathrm{NW}^{1}$, Jegavanthan $\mathrm{A}^{1}$, Hewarathna $\mathrm{UI}^{1}$, Karunaratne RMSP ${ }^{1}$, Siribaddana MAH $^{1}$, Ambagammana DMJMH ${ }^{1}$, Jayawickreme SR ${ }^{1}$

${ }^{1}$ Cardiology Unit, Teaching Hospital, Kandy, Sri Lanka

\section{Key words: Cardiology, ischemic heart disease, Coronary Slow Flow Syndrome, Coronary angiography}

\begin{abstract}
Introduction
Phenomenon of Coronary Slow Flow (CSF) is a rare clinical entity leading to angina, ischemic evidence in electrocardiography and cardiac scintigraphy. The functional and structural effects of CSF on the myocardium are yet to be understood.

Objectives

To evaluate the characteristics of patients with CSF phenomenon (syndrome Y) Methods

Between January 2014 and December 2015, all consecutive patients scheduled for coronary angiography and diagnosed with the CSF phenomenon were included. CSF was diagnosed based on the Thrombolysis In Myocardial Infarction (TIMI) frame count (TFC). A TFC $>27$ indicated the diagnosis of CSF phenomenon.

Results

Among 3250 patients scheduled for selective coronary angiography, 34 (1.05\%) met the CSF criteria. The mean age of the sample was $51.2 \pm 8.8$ years and $61.8 \%$ were males. Risk factors were diabetes mellitus (11.8\%), hypertension (41.2\%) and dyslipidemia (20\%) while presentation was stable angina $(45 \%)$ and acute coronary syndromes (55\%). The mean ejection fraction of the sample was $55.9 \pm$ $8.9 \%$. Prevalence of CSF in triple vessels was $35.5 \%$. There were $20.6 \%$ with slow flow in Left Anterior Descending (LAD) \& Left Circumflex (LCX), $23.5 \%$ with slow flow in LAD \& Right Coronary Artery (RCA) and $2.9 \%$ with slow flow in LCX \& RCA. The LAD was involved in $97 \%$ of cases, whereas RCA and LCX were involved in $61.8 \%$ and $58.8 \%$ of cases respectively. Isolated LAD CSF involvement was observed in $17.6 \%$ patients. The mean corrected TFC of LAD was $45.7 \pm 11.8$.

Conclusion

CSF phenomenon is an uncommon finding in patients with angina. Triple vessel involvement is seen more frequently among the affected and LAD is the often involved vessel. This highlights the need of further exploration pertain to the clinical cause of these patients in future follow-up studies.
\end{abstract}

